\section{A E E T}

ASOCIACIÓN ESPAÑOLA DE ECOLOGÍA TERRESTRE

\title{
La investigación y seguimiento ecológico a largo plazo (LTER)
}

\author{
R. Díaz-Delgado ${ }^{1, *}$ \\ (1) Estación Biológica de Doñana-CSIC. Avda. Américo Vespucio s/n. 41092 Sevilla. España. \\ * Autor de correspondencia: R. Díaz-Delgado [rdiaz@ebd.csic.es]
}

> Recibido el 18 de marzo de 2016 - Aceptado el 03 de abril de 2016

Díaz-Delgado, R. 2016. La investigación y seguimiento ecológico a largo plazo (LTER). Ecosistemas 25(1): 01-03. Doi.:

10.7818/ECOS.2016.25-1.01

\section{Una cuestión de escalas}

Desde los comienzos de la ecología, la mayor parte de los trabajos científicos experimentales han sido efectuados durante periodos cortos de tiempo y a escala local (Callahan 1984, Tilman 1989, Müller et al. 2010a). Esto se ha debido fundamentalmente a las limitaciones temporales en la financiación de los proyectos de investigación, normalmente de corta duración (3-4 años) o bien a la disponibilidad de los propios investigadores para poder continuar con sus estudios a medio y largo plazo. En consecuencia, los cambios a largo plazo han sido con frecuencia ignorados en los trabajos de ecología (Hobbie et al. 2003).

En las últimas décadas, el ritmo característico de los procesos de cambio climático y cambio global ha contribuido definitivamente a cambiar las escalas de aproximación a estas cuestiones empleadas por la comunidad científica. La observación y seguimiento a largo plazo de variables de interés para la ecología deviene así indispensable hoy en día para la detección de tendencias y cambios de relevancia para los ecosistemas del planeta. La reducción de la superficie forestal a escala global, los procesos de eutrofización y contaminación, los efectos potenciales de las especies invasoras en los ecosistemas y en los servicios que proveen, son solo algunos de los ejemplos en los que las tasas de cambio son lentas. A la hora de plantear proyectos de restauración o de realizar tomas de decisiones en conservación, resulta esencial poder contar con información de base, datos de referencia para poder elaborar los análisis temporales y las predicciones con mayor solidez estadística. También, las observaciones a largo plazo son necesarias para responder a cuestiones teóricas de la ecología, tales como reducir las incertidumbres sobre la irreversibilidad de los cambios estructurales de los ecosistemas, el éxito de los programas de restauración, conservación y gestión de espacios protegidos, y mejorar la definición de las propiedades emergentes de los ecosistemas como la resiliencia, la estabilidad y la adaptabilidad. Éste es el marco contextual de trabajo de la investigación ecológica a largo plazo (LongTerm Ecological Research, LTER).

\section{El ecosistema como objeto focal de estudio}

Dada la magnitud de los cambios planetarios el objeto de estudio de la investigación ecológica a largo plazo suele ser el ecosistema. La complejidad inherente de esta unidad de estudio ha sido abordada mediante la aproximación reduccionista, la teoría de sistemas y recientemente mediante la incorporación de los vínculos entre los compartimentos ecológico, social y humano (socio-ecología). Estudios ecológicos a largo plazo han permitido evidenciar las relaciones entre la biodiversidad y el funcionamiento del ecosistema (Schulze y Mooney 1994) y han sido aplicados para mejorar el conocimiento de los ciclos de nutrientes, los humedales, los ecosistemas marinos y forestales, entre otros (Müller et al. 2010b). Schöentaler et al. (2003) identifican varios puntos comunes en los estudios ecológicos a largo plazo:

- consideración de los efectos indirectos, crónicos y deslocalizados;

- integración de los procesos ecológicos y su relación con la gestión ambiental;

- combinación de balances hídricos, ciclos de nutrientes, intercambio de materia y energía con la dinámica de comunidades;

- consideración de la auto-organización como un proceso básico del ecosistema;

- agregación de las componentes funcional y estructural del ecosistema;

- concienciación de la irreversibilidad de los cambios en las funciones y estructura de los ecosistemas;

- uso de múltiples escalas y resoluciones en términos de espacio, tiempo, disciplinas y profundidad analítica.

Todos ellos requieren del empleo de largas series temporales de datos. La denominada ciencia del ecosistema se nutre de ellas para modelar y simular la respuesta de los ecosistemas a los cambios inducidos por la humanidad. Además, existe un uso generalizado del concepto de ecosistema en la legislación nacional e internacional reconociendo las escalas temporales intrínsecas, la inevitabilidad del cambio y la necesidad de una planificación a largo plazo en la gestión de los ecosistemas. En este sentido, los programas de seguimiento son esenciales tanto como sistemas de alerta temprana, como proveedores de series temporales de datos ecológicos que proporcionen la línea de base en la toma de decisiones.

\section{Compartiendo los datos y la información}

Este aumento de las escalas temporales y espaciales en la investigación ecológica conlleva además una mejora reclamada en 
la eficiencia en el uso de los datos así adquiridos más allá del uso concreto por su investigador propietario. Es ésta una de las propiedades emergentes que surgen de las redes de investigación ecológica a largo plazo (ILTER, LTER-Europa, LTER-España). En otras palabras, el gran esfuerzo efectuado por las administraciones competentes y por la comunidad científica en establecer redes de investigación ecológica a largo plazo no solo ha redundado en un reenfoque metodológico y en un incremento de series temporales de datos ecológicos, sino también en la puesta en marcha de procesos de armonización y consenso sobre protocolos, indicadores y variables ecológicas a escala internacional (ver ECOPAR: Parameters and Methods for Ecosystem Research and Monitoring y Handbook of standardized ecosystem protocols), o en la adopción de estándares de metadatos ecológicos y herramientas comunes de edición, almacenamiento y consulta de metadatos de los sitios de la red LTER-Europa y la red internacional ILTER. La gestión e integración de la información deviene así para la investigación ecológica a largo plazo un prerrequisito esencial para poder abordar sus objetivos científicos (Bonet y San Gil 2010).

\section{Contribución científica}

Es habitual citar como uno de los estudios ecológicos a largo plazo pioneros él de Rothamsted descrito en (Taylor 1989) iniciado en 1843. Este estudio evidenció la limitación por nitrógeno en el crecimiento de las plantas. Desde aquel momento diferentes iniciativas comenzaron a establecer sitios experimentales y a proporcionar series de datos ecológicos de gran alcance científico en ecología tales como los proporcionados por el sitio LTER de la cuenca forestal de Hubbard Brook (Likens 1977). Evidentemente, el ritmo de publicación de la investigación ecológica a largo plazo no es comparable con el de otros ámbitos científicos. No obstante, el número de publicaciones en este ámbito ha ido creciendo de forma considerable (Fig. 1). Las diferentes áreas de conocimiento indican la transdisciplinariedad de estos estudios (Fig. 1), y se destaca la importante contribución española y el liderazgo estadounidense.

Como gran valor añadido, el trabajo en red permite investigaciones que no pueden llevarse a cabo a escala de parcela evidenciando patrones globales. Un ejemplo de ellos es el experimento de descomposición de hojarasca -Long-Term Inter-site Decomposition Experiment- (Adair et al. 2008, Currie et al. 2010) en el que la liberación de nitrógeno en el suelo se explica en todos los sitios muestreados por los dos mismos factores: la concentración inicial de nitrógeno y la cantidad de materia orgánica remanente. Ejemplos de este tipo de contribuciones no solo tienen lugar en Estados Unidos. El reciente trabajo publicado por Dirnböck et al. (2014) resume los efectos generalizados de la fertilización atmosférica por deposición de nitrógeno sobre las comunidades de plantas de sotobosque a escala Europea. Este trabajo representa una prueba de concepto del funcionamiento de la red LTER-Europa: acogiendo las iniciativas científicas, ofreciendo la red de sitios (ecosistemas), facilitando las series temporales de datos y apoyando a los investigadores mediante accesos, reuniones, propuestas de financiación, etc.

En definitiva, la investigación ecológica a largo plazo se ha consolidado en el ámbito académico y ha conseguido despertar el interés de la comunidad científica que trabaja en ecología no solo por la publicación de sus hallazgos si no también por el carácter colaborativo y transdisciplinar de sus actividades. En el caso español desde la creación de la red LTER-España en 2008 (Díaz-Delgado 2010) los diferentes nodos, todos ellos espacios protegidos, han ido consolidando esta aproximación incorporando en su misión la implementación de programas de seguimiento ambiental a largo plazo (Zamora 2010) y su compromiso con la gestión de estos espacios protegidos (para más detalles ver Ecosistemas 19, Vol. 2).

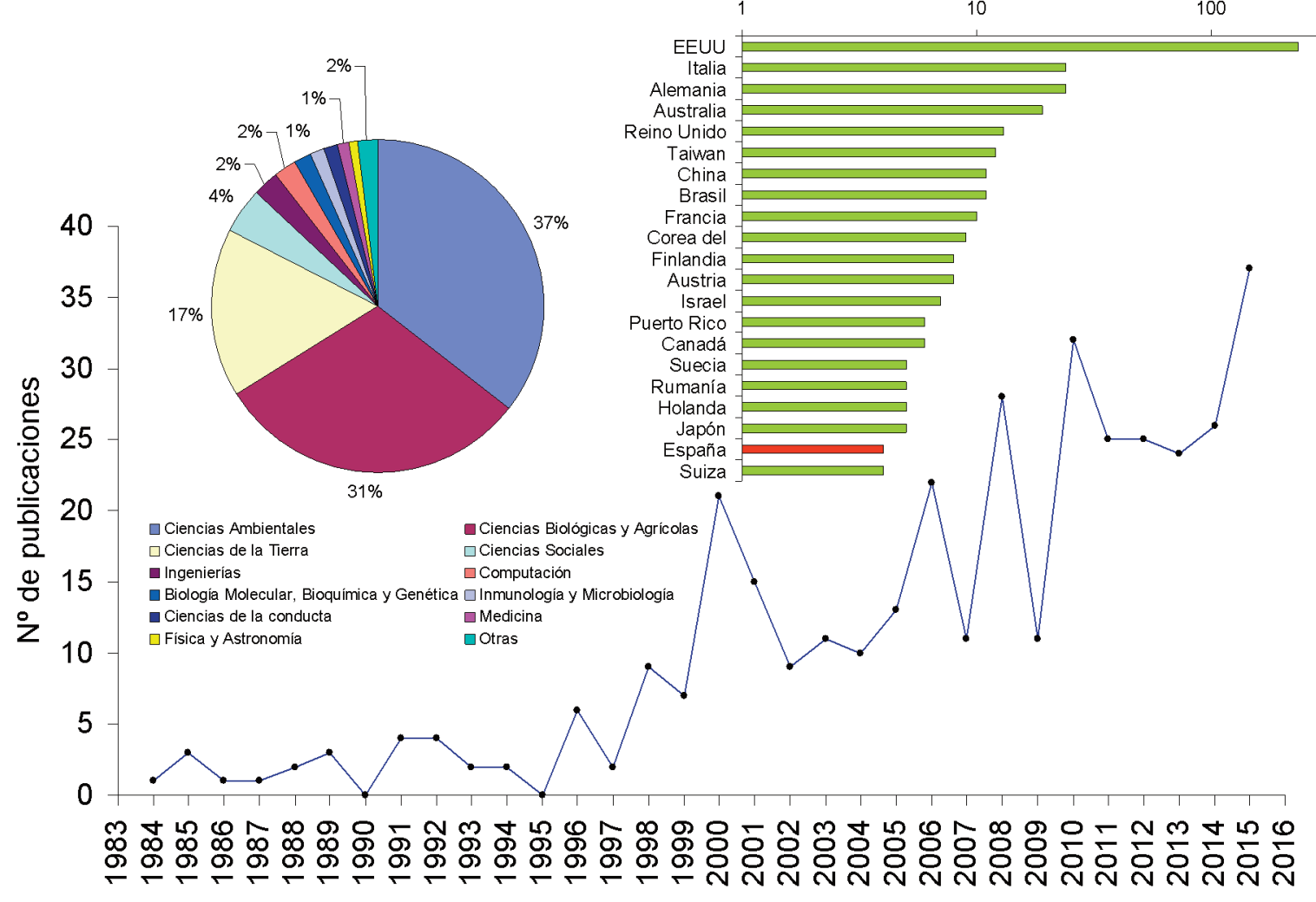

Figura 1. Número de publicaciones científicas (revistas SCI, congresos internacionales y libros) en los últimos 30 años sobre LTER. Se muestra también el número de contribuciones por países y las áreas temáticas de las publicaciones. Datos obtenidos sobre búsqueda en Scopus con las palabras clave "LTER" Y "Long-term ecological" O "Long-term ecosystem" O "Long-term monitoring" en los campos título, resumen o palabras clave.

Figure 1. Number of scientific publications (SCl journals, international conferences and books) in the last 30 years about LTER topic. The figure also shows the number of contributions per country and the thematic areas covered by the publications. Results from Scopus search using the keywords "LTER" AND "Long-term ecological" OR "Long-term ecosystem" OR "Long-term monitoring" in the fields title, abstract or keywords. 


\section{Investigación y seguimiento ecológico a largo plazo en España y Argentina}

El presente monográfico muestra los avances en la implementación de la investigación ecológica a largo plazo en algunos de los sitios que forman parte de la red española y un caso concreto en el Sur de Argentina. Se presentan un total de siete trabajos, cinco de ellos de revisión sobre la creciente contribución científica surgida de esta aproximación en tres Parques Nacionales: Tablas de Daimiel (Sánchez-Carrillo et al. 2016), Doñana (Díaz-Delgado et al. 2016) y Ordesa y Monte Perdido (García et al. 2016); en la Red de Seguimiento de Cambio Global en Parques Nacionales (Bonache et al. 2016) y uno en Argentina. Los tres parques nacionales mantienen su constancia en la adquisición sistemática de información de carácter ecológico con una gran vocación en los programas de seguimiento a largo plazo y en la aportación de herramientas para la toma de decisiones en la gestión de estos espacios protegidos. Como artículos de investigación se presentan los trabajos efectuados en el Montseny evidenciando la pérdida en la diversidad de aves por abandono rural (Herrando et al. 2016) y los efectos del cambio global sobre la productividad de los melojares y las comunidades de aves en el Parque Nacional de Sierra Nevada (PérezLuque et al. 2016). Finalmente, se presenta se presenta el trabajo de revisión de la fabulosa labor emprendida en la Patagonia Austral para establecer una red de sitios de investigación ecológica a largo plazo y los resultados obtenidos en diferentes ámbitos de investigación (Martínez-Pastur et al. 2016).

Es para los autores un placer poder contribuir a la visibilidad de estos trabajos y esperamos sinceramente sean del agrado de los lectores de Ecosistemas.

\section{Agradecimientos}

La red LTER-España ha mejorado su integración y participación en las redes de investigación ecológica a largo plazo gracias a financiación nacional del proyecto ACl-Comités (ACl2008-0815) del Programa de Internacionalización de la I+D del antiguo Ministerio de Ciencia e Innovación, y europea mediante el programa LIFE+ a través del proyecto EnvEurope (Environmental quality and pressures assessment across Europe: the LTER network as an integrated and shared system for ecosystem monitoring, LIFE08 ENV/IT/000399) y del proyecto EXPEER (Experimentation in Ecosystem Research) del $7^{\circ}$ Programa Marco. En la actualidad la red LTER-España participa en el proyecto eLTER (European Long-Term Ecosystem and Socio-Ecological Research Infrastructure) del programa H2020 (Infraia), que sostiene el desarrollo continuado de la red europea y posibilita la investigación en un total de 18 sitios de la red mediante un programa de accesos financiados de investigación (http://www.ltereurope.net/elter/ta). La Fundación Biodiversidad financia el proyecto "Coordinación de las acciones de seguimiento del Cambio Global en la Red LTER España desde 2015" que promueve el desarrollo de la red LTER-España.

\section{Referencias}

Adair, E.C., Parton, W.J., del Grosso, S.J., Silver, W.L., Harmon, M.E., Hall, S.A., Burke, I.C. Hart, S.C. 2008. Simple three-pool model accurately describes patterns of long-term litter decomposition in diverse climates. Global Change Biology 14(11):2636-2660.

Bonache, J., de Mingo-Sancho, G., Serrada, J., Amengual, P., Perales, J., Martínez, R., Rodado, S., Albornos, E. 2016. El seguimiento y la evaluación a largo plazo en la Red española de Parques Nacionales. Ecosistemas 25(1): 31-48. Doi.: 10.7818/ECOS.2016.25-1.05

Bonet, F., San Gil, I. 2010. Gestión de la información ambiental en los espacios protegidos y en las redes de seguimiento del cambio global. Ecosistemas 19(2): 84-96.

Callahan, J.T. 1984. Long-Term Ecological Research. BioScience 34(6):363367.
Currie, W.S., Harmon, M.E., Burke, I.C., Hart, S.C., Parton, W.J., Silver, W. 2010. Cross-biome transplants of plant litter show decomposition models extend to a broader climatic range but lose predictability at the decadal time scale. Global Change Biology 16(6):1744-1761.

Díaz-Delgado, R. 2010. Puesta a punto e implementación de un programa integrado de seguimiento ecológico a largo plazo en el Espacio Natural de Doñana. Ecosistemas 19(2): 42-55.

Díaz-Delgado, R., Carro, F., QuirósF., Osuna, A., Baena, M. 2016. Contribución del seguimiento ecológico a largo plazo a la investigación y la gestión en la plataforma LTSER-Doñana. Ecosistemas 25(1): 09-18. Doi.: 10.7818/ECOS.2016.25-1.03

Dirnböck, T., Grandin, Bernhardt-Römermann, M., Beudert, B., Canullo, R., Forsius, M., et al. 2014. Forest floor vegetation response to nitrogen deposition in Europe. Global Change Biology 20(2):429-440.

García, M.B., Alados, C.L., Antor, R., Benito Alonso, J.L., Camarero, J.J., Carmena, F., Errea, P., Fillat, F., García-González, R., García-Ruiz, J.M., Gartzia, M., Gómez García, D., Gómez, I., González-Sampériz, P., Gutiérrez, E. Jiménez, J.J., López-Moreno, J.I., Mata, P., Moreno, A., Montserrat, P., Nuche, P., Pardo, I., Revuelto, J., Rieradeval, M.I., Sáiz, H., Tejero, P., Vicente-Serrano, S., Villagrasa, E., Villar, I., Valero-Garcés, B. 2016. Integrando escalas y métodos LTER para comprender la dinámica global de un espacio protegido de montaña: el Parque $\mathrm{Na}$ cional de Ordesa y Monte Perdido. Ecosistemas 25(1): 19-30. Doi.: 10.7818/ECOS.2016.25-1.04

Herrando, S., Anton, M., Brotons, LI., Guinart, D. 2016. La pérdida de biodiversidad por abandono rural en el LTER Montseny cuantificada a partir del monitoreo de aves. Ecosistemas 25(1): 58-64. Doi.: 10.7818/ECOS.2016.25-1.07

Hobbie, J.E., Carpenter, S.R., Grimm, N.B., Gosz, J.R., Seastedt, T.R. 2003. The US Long Term Ecological Research Program. BioScience 53(1):21-32.

Likens, G.E. 1977. Biogeochemistry of a Forested Ecosystem. Springer Science and Business Media, Nueva York, Estados Unidos.

Martínez-Pastur, G., Peri, P.L., Lecinas, M.V., Soler, R., Bahamonde, H.A., Valenzuela, A.E.J., Cabello, J.L., Anderson C.B. 2016. Investigación socio-ecológica a largo plazo en la Patagonia Austral: Estrategias interdisciplinarias para lograr la conservación de los recursos naturales a través de un manejo sustentable bajo escenarios de cambio global. Ecosistemas 25(1): 49-57. Doi.: 10.7818/ECOS.2016.25-1.06

Müller, F., Baessler, C., Schubert, H., Klotz, S. 2010a. Long-Term Ecological Research: Between Theory and Application. Springer Science and Business Media, Dordtretch, Alemania.

Müller, F., Gnauck, A., Wenkel, K.-O., Schubert, H., Bredemeier M. 2010b. Theoretical Demands for Long-Term Ecological Research and the Management of Long-Term Data Sets. En: Müller, F., Baessler, C. Schubert, H., Klotz, S. (eds.), Long-Term Ecological Research, Between Theory and Application pp. 11-25. Springer Science and Business Media, Dordtretch, Alemania.

Pérez-Luque, A.J., Bonet, F.J., Zamora, R., Bara-Azcón, J.M., Aspizua, R., Sánchez-Gutiérrez, F.J. 2016. Señales del cambio global en el sitio LTERSierra Nevada. Ecosistemas 25(1): 65-71. Doi.: 10.7818/ECOS.2016.251.08

Sánchez-Carrillo,S., Álvarez-Cobelas, M., Cirujano, S., Carrasco-Redondo, M., Díaz-Cambronero, A. 2016. La información a largo plazo como herramienta clave para la evaluación de los cambios ambientales en las tablas de Daimiel: LTER-Daimiel. Ecosistemas 25(1): 04-08. Doi.: 10.7818/ECOS.2016.25-1.02

Schoenthaler, K., Mueller, F. Barkmann, J. 2003. Synopsis of system approaches to environmental research - German contribution to ecosystem management. UBA-Texte 85/03:1-142. Disponible en www.umweltbundesamt.de/sites/default/files/medien/publikation/long/2437.pdf

Schulze, E.-D., Mooney, H.A. 1994. Biodiversity and Ecosystem Function. Springer Berlin Heidelberg, Berlin, Alemania.

Taylor, L.R. 1989. Objective and Experiment in Long-Term Research. En: Likens, G.E. (ed.), Long-Term Studies in Ecology, pp. 20-70. Springer, Nueva York, Estados Unidos.

Tilman, D. 1989. Ecological Experimentation: Strengths and Conceptual Problems. En: Likens, G.E. (ed.), Long-Term Studies in Ecology, pp. 136-157. Springer, Nueva York, Estados Unidos.

Zamora, R. 2010. Las Áreas protegidas como Observatorios del Cambio Global. Ecosistemas 19(2): 1-4. 